\title{
Canu Roland, Chaulet Johann, Datchary Caroline et Figeac Julien (dir.) : Critiques du numérique
}

\section{Kaltoum Mahmoudi}

\section{(2) OpenEdition}

\section{Journals}

Édition électronique

URL : https://journals.openedition.org/edc/10466

DOI : $10.4000 /$ edc. 10466

ISSN : 2101-0366

Éditeur

Université de Lille

Édition imprimée

Date de publication : 1 juillet 2020

Pagination : 205-208

ISBN : 978-2-917562-23-9

ISSN : $1270-6841$

Référence électronique

Kaltoum Mahmoudi, «Canu Roland, Chaulet Johann, Datchary Caroline et Figeac Julien (dir.) :

Critiques du numérique », Études de communication [En ligne], 54 | 2020, mis en ligne le 01 juillet 2020, consulté le 07 janvier 2022. URL : http://journals.openedition.org/edc/10466 ; DOI : https://doi.org/ 10.4000/edc. 10466

Ce document a été généré automatiquement le 7 janvier 2022

(c) Tous droits réservés 


\title{
Canu Roland, Chaulet Johann, Datchary Caroline et Figeac Julien (dir.) : Critiques du numérique
}

\author{
Kaltoum Mahmoudi
}

\section{RÉFÉRENCE}

Canu R., Chaulet J., Datchary C. et Figeac J. (dir.) (2018). Critiques du numérique, Paris, L'Harmattan.

Dans

la

collection

« Humanités

numériques

» dirigée par Julien Longhi paraît ce recueil de huit contributions centrées sur les rapports entre activités critiques et technologies numériques à travers le discours des

acteurs. L'ouvrage fait suite aux travaux du séminaire PragmaTIC (2016-2017) organisé par l'université de Toulouse $2^{1}$. La moitié des auteurs du recueil est issue du champ de la sociologie. Coordonné par deux enseignants chercheurs en sociologie (Roland Canu et Caroline Datchary) et deux sociologues chargés de recherche au CNRS (Johann Chaulet et Julien Figeac), l'ouvrage défend pour autant une approche transversale et transdisciplinaire.

2 L'introduction présente successivement l'ensemble des contributions organisées autour de trois parties équilibrées. Ce plan annoncé n'est cependant pas apparent à travers le sommaire. Chaque contribution est introduite par un préambule facilitant ainsi l'entrée du lecteur dans le sujet. Leur mise en perspective révèle qu'autour du numérique s'articule un double rapport à la critique : le numérique est objet de critiques et outil de propagation des critiques. Cette double perspective est le fil rouge du recueil qui s'achève sur la contribution de Dominique Boullier dont l'intérêt est de regrouper l'ensemble des textes autour d'une typologie de la critique offrant ainsi au lecteur un cadre théorique à partir d'une approche plurielle. 
3 De quoi le numérique est-il la critique ? Cette première partie du recueil réunit deux contributions complémentaires qui ont en commun d'étudier les critiques des salariés liées aux conséquences de l'introduction des technologies numériques. L'analyse des trajectoires de ces critiques est proposée par Moustafa Zouinar. Il constate une porosité entre vie professionnelle et vie privée (joignabilité illimitée, pression temporelle...) qui pousse ainsi les salariés à déployer des stratégies individuelles et collectives de régulation, de résistance et de contrôle des outils numériques notamment à travers des pratiques de déconnexion. Le numérique est également l'objet de la critique de Nikos Smyrnaios. La concentration oligopolistique de l'Internet impose selon lui des logiques hétéronomes aux journalistes et éditeurs de sites d'information en ligne. À partir d'une approche socio-économique, il dénonce l'émergence de relations de dépendance inégalitaire entre journalistes et infomédiaires qui menacent les sociétés démocratiques libérales.

4 Logiques hétéronomes versus logiques d'empowerment. Comment les acteurs sociaux s'approprient-ils les potentialités offertes par les technologies numériques pour développer leurs activités et postures critiques? Les trois contributions de cette seconde partie apportent tout d'abord des éléments de réponse à travers l'analyse de pratiques collectives de nature contestataire et ont le mérite ensuite de mettre en lumière différents répertoires de l'expression critique. Loin des démocraties libérales, Marie Laure Geoffray amène le lecteur à adopter un regard global à travers le cas du régime autoritaire de Cuba. La libéralisation des usages du numérique est un facteur d'émancipation qui n'a pas pour conséquence une politisation plus forte des citoyens cubains mais qui les autorise à défier les formes autoritaires de la domination. A contrario, les deux contributions suivantes dévoilent que la participation collective aux débats citoyens via les espaces de discussion en ligne, oriente l'action politique. Valérie Beaudouin se saisit du cas d'une stèle funéraire nommée "Boyau Eugène» dont l'abandon par les pouvoirs publics suscite de nombreuses critiques de la part de passionnés amateurs de la Grande Guerre qui revendiquent la préservation de la mémoire contre l'oubli. Dans un registre tragico-émotionnel, les réactions sur YouTube provoquées par les fusillades dans deux établissements scolaires américains (school shooting) ont éveillé une participation active aux divers débats dont celui sur le contrôle des armes (gun control). L'originalité de la contribution de Nathalie Paton et de Julien Figeac est double: elle réside en l'émergence d'une pluralité de répertoires de l'expression critique à travers les désaccords entre internautes d'une part, et tient à l'ajout d'images extraites des corpus de recherche d'autre part. S'exprimer à visage découvert demeure une règle de sociabilité sur ces espaces de discussion collective qui produisent en fait leurs normes et règles d'échange propres.

5 La dernière partie du recueil réunit trois contributions de chercheurs issus du domaine de la sociologie et centrées sur les postures critiques occupées par les acteurs qui s'approprient les potentialités contestataires des TIC. À partir de résultats d'observations menées in situ dans le cadre des mouvements sociaux des Indignés (Barcelone, 2011) et de Nuit debout (Toulouse et Paris, 2016), Jérôme Ferret s'empare de la question de la réputation. La production et la diffusion de vidéos produites en immersion donnent lieu à différentes formes de "médiatisations sauvages " (phénomènes du riot porn et du copwatching) visant à préserver la réputation nonviolente de ces mouvements et à discréditer celle des instances institutionnelles. Au croisement d'une sociologie des cultures numériques et du genre, Jessica Soler-Benonie 
plonge le lecteur dans l'univers des pratiques vidéoludiques à travers l'argumentaire critique de deux bloggeuses féministes passionnées de jeux vidéo portant sur les représentations sexistes et stéréotypées et les mécanismes de la domination masculine. Leurs procédés narratifs et les commentaires que leurs critiques suscitent sont analysés dévoilant ainsi des dynamiques d'empowerment. L'ouvrage se clôt sur une typologie en quatre critiques formulée par Boullier et envisagée sous l'angle des discours et des " machines critiques ». Cette conclusion synthétisante relie toutes les contributions du recueil et ouvre enfin des pistes de réflexion sur l'émergence d'une troisième génération de sciences sociales qui appelle les chercheurs à se saisir de manière critique des questions liées au numérique, dont celle de la traçabilité qui, par ailleurs, selon nous, ne constitue pas l'unique enjeu.

6 À destination des chercheurs, salariés et usagers des technologies numériques, cette contribution collective originale se distingue par le croisement des expériences des acteurs sociaux et des analyses des chercheurs à travers une pluralité de regards et d'approches. Une lecture non linéaire de l'ouvrage est recommandée en fonction des centres d'intérêt de tous ceux qui portent attention aux enjeux du numérique et de ses médiations.

\section{NOTES}

1. https://sms.univ-tlse2.fr/accueil-sms/comunitic/le-seminaire-pragmatic-210884.kjsp

\section{AUTEURS}

\section{KALTOUM MAHMOUDI}

Univ. Lille, EA 4073 - GERiiCO, F-59000 Lille, France

kaltoum.mahmoudi@univ-lille.fr 